\title{
Normal Cloud Distribution probability density fast calculation
}

\author{
Jingfang Wang \\ School of Electrical \& Information Engineering \\ Hunan International Economics University \\ Changsha, China \\ e-mail: matlab_wjf@126.com
}

\begin{abstract}
Normal cloud model with certainty, uncertainty and change into the stable characteristics in the expression of the knowledge, reflects the basic principle of the nature of species evolution, intelligent control, fuzzy evaluation, and other fields has been applied; Normal cloud distribution of the probability density is not an explicit expression, and is a special function integral to its in-depth application difficult; A fast calculating method of normal cloud distribution probability density is designed. According to the distribution characteristics, the sampling point on the logarithmic span is first designed, the second is to use the golden section principle to select the sample points before and after the interval; the method has been applied to the mixed-normal cloud model, the voice model, the effect is very good effect.
\end{abstract}

Keywords- normal cloud distribution; probability density; Numerical integration; golden section; logarithmic sampling

\section{INTRODUCTION}

Normal cloud model is a Chinese scholar Professor Li Deyi [1], the qualitative and quantitative transformation model. With the deepening of the uncertainty, uncertainty is the charm of this world, only the uncertainty itself is OK. In many uncertainties, the randomness and fuzziness is the most basic. Probability theory and fuzzy math, Chinese Academy of Engineering, Professor Li Deyi in 1995 on the basis of probability theory and fuzzy mathematics normal cloud concept, and the fuzziness and randomness and both in dealing with uncertainty lack the correlation between. The cloud model has just a dozen years, has been successfully applied to data mining since Academician Li Deyi et al [2], decision analysis, intelligent control [3,4], system assessment [6] and many other fields. Normal cloud model is the most important kind of cloud model, because of its good mathematical properties can be expressed uncertainty phenomena in the natural sciences, social sciences [8]. Normal cloud model uncertainty with certainty, stability and variations among the characteristics of the knowledge representation, embodies the basic principle of the nature of species evolution.

Central limit theorem explained theoretically produce the conditions of the normal distribution, the simple and intuitive description of the central limit theorem is: If you decide the results of a random variable is a large number of small, independent and random factors, and each random factors separate the role of relatively uniform small, no one can play the leading role of the overriding factors, then the random variable is generally approximately obey the normal distribution. The normal distribution is widely found in natural phenomena, social phenomena, science and technology, and production activities, a lot of random phenomena encountered in practice obedience or approximate normal distribution. Naturally occurring wider than the normal distribution of a random variable distribution, in reality, mainly in a small number of individuals made substantial contribution to the (resource-intensive), such as in air, nitrogen, oxygen: other ratio of 78:21:1, human brain in water and other substances to $80: 20$, most of the oil reserves from less oilfield, the number of major disaster is only a small part of the number of all disaster; intuitive features is the bulk of short, small head, quantity $\rightarrow$ large amount of a single product species less $\rightarrow$ short bulk of the kinds of aspects $\rightarrow$ small amount $\rightarrow$ long small tail varieties; heavytailed distribution means that you can get a great value in a greater probability of randomness and weak, heavy-tailed distribution is generally expressed morbid increase kinds of results are determined to have a heavy-tailed distribution, including income distribution, financial reporting, insurance expenses, page reference links, they are non-stationary processes with heavy-tailed characteristics. Normal cloud distribution has a heavy tail characteristics [7,9], it determines the random phenomenon factors alone, not even small, not independent of each other, there is a certain degree of interdependence, will not be able to comply with the generation of a normal distribution conditions, does not constitute a normal distribution. Academician Li Deyi cloud model to describe this kind of randomness, the expansion of the normal distribution for the Pan-normal, with a new independent parameters - ultra entropy, to measure the degree of deviation from the normal distribution.

In this paper, a normal cloud distribution numerical calculation of the probability density and fast calculation.

\section{NORMAL CLOUD MODEL}

Normal cloud is a the language value uncertainty transformation model between a qualitative concept and its quantitative said. It mainly reflects the uncertainty with certainty, stability and variations among the characteristics of the objective world of things or human knowledge in the concept of two kinds of uncertainty: fuzziness He also vital (the border) and random (the probability of occurrence), and both completely integrated together, constitute the qualitative and quantitative inter mapping. Because the normal cloud in the number of domain space, neither is a certain probability density function is not a clear membership curve, but a 
scalable, no edge, flexible, one pair of myopia boundless distance, like a cloud more mathematical mapping image, have similar uncertain nature and natural phenomena in the cloud borrow "cloud" so named convert this data - the concept of mathematical theory.

Normal cloud model with independent set of parameters to express a qualitative concept of digital features, reflecting the concept of uncertainty. Based on the membership function of the normal distribution function with normality, this set of parameters with expectation Ex, three digital characteristics of the entropy En, hyper entropy He characterized:

Expectation Ex (Expected value) in the domain of space in the most capable of representing the qualitative point of the concept is the concept to quantify the most typical sample point.

Entropy En (Entropy) represents a qualitative concept can measure particle size, usually the the entropy greater the more macro concept. Also a qualitative measure of the uncertainty of the concept of joint decision by the concept of randomness and fuzziness on the one hand En qualitative concept of randomness measure reflects the degree of discrete able to represent the qualitative concept of cloud droplets; the other hand, The qualitative concept also vital He metrics, reflecting the range in the domain of space can be accepted by the concept of cloud droplets.

Hyper entropy He (Hyper entropy) entropy measure of uncertainty, which reflects the randomness of the sample representing the value of the qualitative concept, reveals the fuzziness and randomness associated.

Normal cloud model using three digital feature design specific algorithms construct an obedient Pan-normal distribution of random variables - cloud droplets. Determine the degree of a qualitative concept, cloud droplets in close to expectations probability, and the cloud droplets representative of this qualitative concept; cloud droplets away from the expectations at the low probability of the cloud droplets can The degree of certainty that is representative of the concept is also smaller. Fuzzy randomness associated mapping function to calculate the degree of certainty, exactly described phenomenon. Determine the extent of each cloud droplets are randomly generated, and each cloud droplets on behalf of the qualitative concept is vague, and always in subtle changes. Such a quantitative value belonging to a qualitative concept language uncertain changes in each cloud droplet manifested, perhaps not dramatic impact to the overall characteristics of the cloud, i.e. a particular cloud droplets may be insignificant. However, the overall distribution of characteristics of a certain number of cloud droplets reflected the fuzziness and randomness of cloud map, that is, the overall shape of the cloud reflect the uncertainty in the quantitative value represents a qualitative concept characteristics.

Normal cloud model [7-8]: the probability distribution of cloud droplets in the domain of space, all cloud droplets consisting of a random variableX, the probability density of $\mathrm{X}$ :

$$
f_{X}(x)=\frac{1}{\sqrt{2 \pi}\left|E n^{\prime}\right|} \exp \left(-\frac{(x-E X)^{2}}{2 E n^{\prime 2}}\right)
$$

En' probability density:

$$
f_{E n^{\prime}}(x)=\frac{1}{\sqrt{2 \pi} H e} \exp \left(-\frac{(x-E n)^{2}}{2 H e^{2}}\right)
$$

$\mathbf{X}$ probability density:

$$
f_{X}(x)=f_{E n^{\prime}}(x) \times f_{X}\left(x \mid E n^{\prime}\right)=\int_{-\infty}^{\infty} \frac{1}{2 \pi H e|y|} \exp \left(-\frac{(x-E X)^{2}}{2 y^{2}}-\frac{(y-E n)^{2}}{2 H e^{2}}\right) d y
$$

Cloud droplets $\mathbf{X}$ of expectation $\boldsymbol{E}(\boldsymbol{X})=\boldsymbol{E x}$; variance $\boldsymbol{D}(\boldsymbol{X})=\boldsymbol{E} \boldsymbol{n}^{2}+\boldsymbol{H} \boldsymbol{e}^{2}$. Algorithm generated by the normal cloud cloud droplets, an expectation $\boldsymbol{E x}$, the variance of the the $\boldsymbol{E} \boldsymbol{n}^{2}+\boldsymbol{H} \boldsymbol{e}^{2}$ random variable presented Pan-normal distribution. The parameter He is a measure of the degree of deviation from the normal distribution can be used to reflect the impact of the concept of non-uniformity of the factors in the domain of space, not an independent phenomenon. If $\mathbf{H e}$ $=\mathbf{0}$, for the normal distribution; cloud droplets if $\boldsymbol{E n}=\mathbf{0 , H e}$ $=\mathbf{0}$, cloud droplets always appear at the desired point Ex uncertainty.

Qualitative concept of a consensus, all quantitative (cloud droplets) to determine the degree of the random variable $\mathrm{Y}$, the probability density is a fixed form of three digital characteristics of the cloud model reflects the concept has nothing to do.Under normal cloud generator algorithm, determine the degree of cloud droplets constitute the random variable $\mathrm{Y}$ certainty of each can be seen as a sample is generated by the random variables $Y=\exp \left(-\frac{(X-E X)^{2}}{2\left(E n^{\prime}\right)^{2}}\right)$. The probability density function of cloud droplets $\mathrm{Y}$ to determine the degree:

$$
f_{Y i}(y)=\frac{1}{\sqrt{-\pi \ln y}} \quad 0<y<1
$$

This nature reflects different linguistic values represented by the uncertainty of the qualitative concept, the common cognitive regularity - uncertainty. Normal cloud model reveals the cognitive mechanism: Although the concept of implication in different areas, despite the different people, different periods have different perceptions of the same concept, qualitative concepts into a large number of quantitative cloud droplets, cloud droplets exhibit different degree of certainty, but to form a general consensus, reflected in different people cloud droplets to determine the degree of cognitive rules is consistent with the common 
cognitive mechanism between the different qualitative concept expressed in different languages, values.

\section{NORMAL CLOUD DISTRIBUTION PROBABILITY DENSITY NUMERICAL CALCULATION}

\section{A. Probability density analysis}

NC(EX, En, He), the normal distribution of the probability density formula (1) is a special function of the integrator. Integrand:

$$
g(y, x)=\frac{1}{2 \pi H e|y|} \exp \left(-\frac{(x-E X)^{2}}{2 y^{2}}-\frac{(y-E n)^{2}}{2 H e^{2}}\right)
$$

Take $\quad(\mathrm{EX}, \mathrm{En}, \mathrm{He})=(0,1,0.1)$,

Draw $g(y, 0.5), g(y, 1), g(y, 2)$ curve in Figure 1.

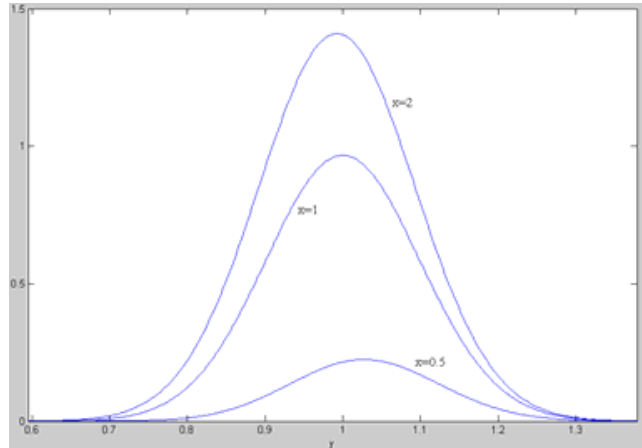

Figure 1. $g(y, x)$ curve of normal cloud distribution density function is integrable functions

The normal distribution $\mathrm{NC}(\mathrm{EX}, \mathrm{En}, \mathrm{He})$ probability density the integrand function $g(y, x)$ amount of information is concentrated in the vicinity of the entropy En. Numerical integration in frequency the En near the sampling whichever is greater, to take a small sampling frequency from En far.

\section{B. The probability density numerical integration design}

Let random variable $\mathrm{X}$ obey the normal cloud distribution NC(EX, En, He), the EX desired, En entropy, He is hyper entropy. then:

1) sampling point design: the interval $(-16,16) \mathrm{K}$ decile, $y<$ En sampling point :

$$
y_{i}=-10^{-16-i \frac{32}{K}}+E n, i=-K,-K+1, \cdots,-2,-1
$$

$y>$ En sampling point :

$$
y_{i}=10^{-16+i \frac{32}{K}}+E n, i=0,1,2, \cdots, K
$$

2) Numerical integration of inter-cell design: $y>E n$,

$$
\Delta_{0}=\Delta_{K}=0, \Delta_{i}=0.618\left(y_{i}-y_{i-1}\right)+0.382\left(y_{i+1}-y_{i}\right), i=1,2, \cdots,(K-1)
$$

$$
y<\text { En }, \Delta_{-i}=\Delta_{i}, i=1,2, \cdots,(K-1), \Delta_{K}=0
$$

3) The probability density of the integrand $g(y, x)$ 's numerical integrator for a fixed value of $x=x_{0}$ :

$$
f\left(x_{0}\right)=\sum_{i=-K}^{K} g\left(y_{i}, x_{0}\right) \Delta_{i}
$$

\section{THE INSTANCE VALIDATION WITH COMPARATIVE} ANALYSIS

Normal distribution $\mathrm{NC}(0,1,0.1)$ of the probability density (1) of symbolic integration MATLAB programs:

\section{$\mathrm{f}=\operatorname{vpa}($ int $(' 1.5924 * \exp (-$ $0.5 / y^{\wedge} 2-50 *(y-$ 1)^2)/abs(y)', -inf, inf))}

Take $x_{0}=1$, regarded as $\mathrm{f}=0.23955$, and that the true value of time-consuming 8.6 seconds.

Numerical integration (3) be regarded as:

$$
f(1)=\sum_{i=-K}^{K} g\left(y_{i}, 1\right) \Delta_{i}=0.23960
$$

The same computer with 0.0301 seconds,

$$
\text { Error }=\frac{|0.23955-0.23960|}{0.23955}=0.0209 \%
$$

And time efficiency of only $0.3 \%$ of year. $\mathrm{NC}(0,1,0.1)$ numerical integration of the probability density see below.

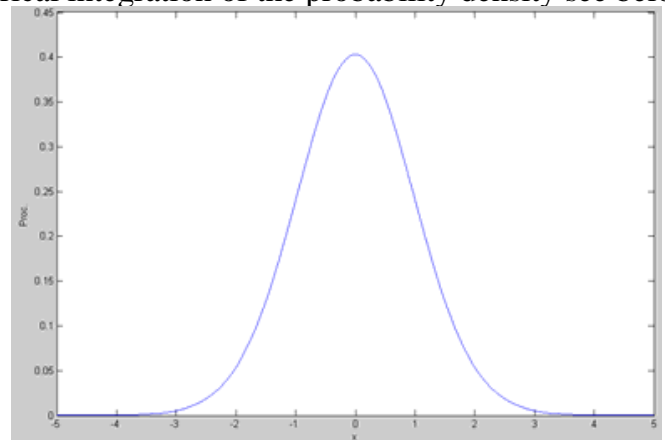

Figure 2. Normal cloud numerical integration of the probability density distribution $\mathrm{NC}(0,1,0.1)$ curve 


\section{CONCLUSIONS}

In this paper, the normal cloud distribution fast calculation of the probability density values, theoretical analysis and experimental simulation. The normal cloud distribution density function is not an explicit expression to make, needed numerical integration, these numerical integration to improve operational efficiency, skill design. Us the digital integrator is used to study the distribution of normal cloud mixed voice modeling, these studies speech enhancement, speech coding, speech recognition, and provide a new theoretical foundation. Normal cloud distribution in the field of artificial intelligence and other applications to provide computing method.

\section{REFERENCES}

[1] Li Deyi Liu Changyu, Du Y, i etal Artificial intelligence with uncertainty [J]. Journal of Softw are, 2004, 15 (11): 1 - 13 (in Chinese)

[2] Wang Shu liang, Li Deren, Shi Wenzhong, etal Cloud model based spatial data min ing $[\mathrm{J}]$. Geographical Information Science, 2003, 9 (2): 67 - 78

[3] Zhang Fei Zhou, Yue-Zu Fan, Shen Cheng Chi using cloud models to achieve intelligent control inverted pendulum [J], Control Theory and Applications, 2000, 17 (4) :519-523

[4] Li Tao, Sun Qu, based on the decoupling vector cloud model smart controller design [J], Journal of Sichuan University (Engineering Science Edition), 2009,41 (5) :232-236

[5] Liu Changyu, Li deyi.Some Statistical Analysis of the Normal Cloud Model [J]. Information and Control, 2005, 34 (2): 236 - 240,

[6] Lu Huijun, Wang Ye, Li Deyi The application of backward cloud in qualitative evaluation [J]. Chinese Journal of Computers, 2003, 26 (8): 1009-1014 (in Chinese)

[7] Li Deyi Liu Changyu, Gan Wen Yan. Heavy tail nature of normal cloud model proved [J]. China Engineering Science, 2011,13 (4) :2023

[8] Li Deyi, Liu Changyu. On normal cloud model resistance [J]. China Engineering Science, 2004,6 (8) :28-34. . 